\title{
Structure of the low-latitude magnetopause: MAGION-4 observations
}

\author{
Z. Nemecek ${ }^{1}$, A. Fedorov ${ }^{2}$, J. Safrankova ${ }^{1}$, G. Zastenker $^{2}$ \\ ${ }^{1}$ Faculty of Mathematics and Physics of Charles University, Prague, Czech Republic \\ ${ }^{2}$ Space Research Institute, Academy of Sciences of Russia, Moscow, Russia
}

Received: 12 December 1995 / Revised: 14 October 1996 Accepted: 16 October 1996

\begin{abstract}
The aims of this paper are (1) briefly to describe the plasma devices onboard the MAGION-4 satellite, launched on 3 August 1995, of the INTERBALL project, and (2) to discuss first observations made near the magnetopause region. During the presented boundary crossings the MAGION-4 observed quasiperiodic pulses of magnetosheath-like plasma in a region of low plasma density. This region is located just earthwards of the magnetopause and is populated by a plasma which, except for the density, has the same parameters as in the magnetosheath. Deeper in the magnetosphere, the encounter of a layer of hot electrons and high-energy ions was interpreted as low-latitude boundary layer.
\end{abstract}

\section{Introduction}

The Earth's magnetopause lies at the locus of points where magnetosheath and magnetospheric pressures balance. Since the magnetosheath pressure at the subsolar point is proportional to the solar-wind dynamic pressure (Landau and Lifshitz, 1959), observations of the solar-wind dynamic pressure can be used to predict magnetopause motion. A number of recent studies (Sibeck et al., 1989 a,b; Potemra et al., 1989) have demonstrated that brief, sharp increases in the solarwind dynamic pressure, each lasting from 1-10 min, are common just upstream of the bow shock. Such pulses are often quasi-periodic with recurrence time-scales of 5-15 min. Their origin remains unknown, although sources in the solar wind and at the quasi-parallel bow shock have been suggested (Sibeck et al., 1989 a,b). They must be considered as a major source of transient magnetopause motion.

The observations made by ISEE $1 / 2$ at the northerndawn magnetopause were interpreted as evidence of the diffusive entry of magnetosheath plasma into the magnetosphere and of Kelvin-Helmholtz instability at the inner edge of the Low-latitude boundary layer (LLBL)
(Sckopke et al., 1981). They have also been interpreted by different authors as indicating magnetic merging and the formation of twisted ropes of interconnected magnetosheath and magnetospheric magnetic lines (Paschmann et al., 1982; Cowley, 1982, 1984, 1986; Saunders, 1983). The flux tube or rope develops both for stationary and non-stationary conditions in the diffusion region (the region where the magnetic field is not frozen into the plasma). Except for singular cases, these flux tubes move along the magnetopause and therefore represent transient events for any fixed location. The formation of such magnetic flux tubes requires the onset of magnetic reconnection or a strong intensification of the reconnection rate.

An alternative mechanism for transient magnetic perturbations is a wavy motion of the magnetopause boundary layer driven by fluctuations of the solar-wind dynamic pressure (Sibeck, 1992, 1993). Sibeck et al. (1990) showed that FTEs [flux transfer events (Russell and Elphic, 1978)] are fully consistent with the properties of magnetosheath magnetic field line draping in the plasma depletion layer, and they presented a model for the signatures of magnetopause motion driven by such solar-wind dynamic-pressure variations. Figure 1 shows a cross-section of the magnetopause in the plane parallel to the ecliptic plane and indicates the expected magnetopause response to a brief increase in the solar-wind dynamic pressure. Such an increase corresponds to an enhancement in both magnetosheath thermal and magnetic pressures which in turn compress the magnetopause locally to form a trough. As the trough approaches a spacecraft in the magnetosphere, that spacecraft observes an increase in the magnetic-field strength. If the spacecraft, exits the magnetosphere and enters the magnetosheath, it should observe streaming energetic ions, a colder, denser, antisunward flowing plasma, and a magnetosheath magnetic-field orientation in the plasma depletion layer.

The scheme of the magnetopause structure depicted in Fig. 1 is widely accepted, but the criteria for the identification of the magnetopause layers can vary in 


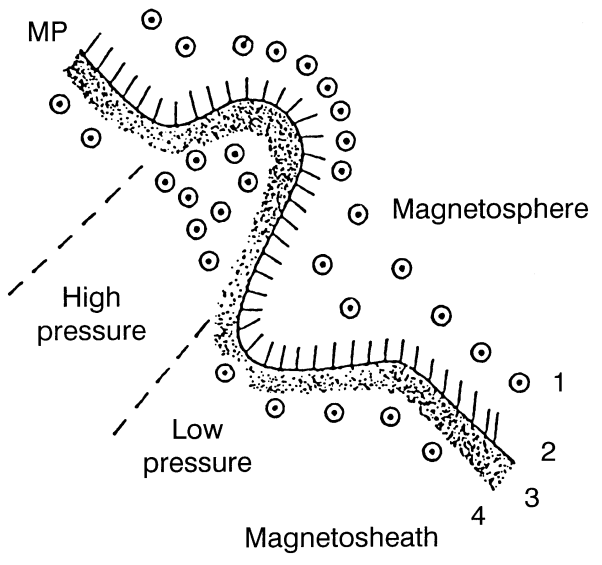

Fig. 1. The wavy downside magnetopause boundary. The magnetosphere is labelled 1, the low-latitude boundary layer as 2, the plasma depletion layer as 3, and the magnetosheath as 4 (from Sibeck et al. 1990)

different papers. Russell (1995) shows that for northward-oriented magnetic field the plasma depletion layer (sheath transition layer) is separated from the magnetosheath by a current layer which causes the magneticfield rotation towards the magnetospheric orientation. The plasma depletion layer itself is characterized by the gradual decrease in the plasma density. This layer is bounded by the other current layer on its inner edge from the LLBL. The LLBL is populated by plasma with the temperature and density intermediate between those of the magnetosheath and the magnetosphere, and the magnetic field has magnetospheric orientation. The boundary between the LLBL and magnetosphere has no signature in the magnetic field and can be identified by the fast increase in the plasma temperature.

During the periods of the southward magnetic field the first turn in the magnetic field which divides the magnetosheath and magnetopause layers is not followed by the decrease in the plasma density. The LLBL is characterized by the high temperature and magnetospheric magnetic field. Phan and Paschmann (1996) introduces the magnetic shear (the angle between magnetic-field vectors inside and just outside the magnetopause) for the classification of the magnetopause crossings. This classification respects the fact that the IMF can change its direction in front of the magnetopause due to the turbulent motion of the magnetosheath plasma. In general, the low-shear magnetopause structure corresponds to that observed during the southward period of the IMF, and the high-shear magnetopause is similar to that observed if the IMF is southward. Phan and Paschmann (1996) define the magnetopause as the beginning of the magnetic-field rotation and the inner edge of the LLBL as the point where the plasma density decreases to $5 \%$ of the magnetosheath value.

The present paper introduces the measurements in the magnetopause region performed by a set of plasma instruments onboard the MAGION-4 INTERBALL subsatellite. The set consists of two experiments: the VDP-S plasma sensor is dedicated to the fast determi- nation of the ion- and electron-flux vector. The MPS/ SPS part measures the electron- and ion-energy distribution function in several directions. Based on the data collected so far and supplemented by the magnetic-field measurement we try to identify the structure of the dawn low-latitude magnetopause layers and to estimate the origin of different plasma populations found in these layers.

\section{Instrumentation}

This section describes the plasma devices placed onboard the MAGION-4 satellite in detail; such a description cannot be found elsewhere, the information given in Triska et al. (1995) being very brief. The INTERBALL project is predominantly dedicated to the study of the Earth's magnetotail, but the satellite spent a long time in the solar wind and the magnetosheath. Thus the plasma devices should cover a wide range of measured quantities.

The main portion of the solar-wind energy is represented by the ion bulk velocity, and thus precise and fast measurement of the ion-flow parameters is required. On the other hand, inside magnetospheric boundaries and in the magnetotail, the hydrodynamic approximation cannot be used, and detailed knowledge of the ion- and electron-energy distribution is of ultimate importance. The VDP-S - MPS/SPS complex is supposed to fulfil all the requirements mentioned; the designs are based on experiments previously flown onboard Prognoz-type satellites: the MONITOR experiment onboard the Prognoz-8 (Zastenker et al., 1982; Avanov et al., 1985) and the BM device which was part of the BIFRAM spectrometer onboard the Prognoz-10 satellite (Bedrikov et al., 1985). The main idea is that for different scientific tasks, different types of sensors are used. This approach simplifies each sensor design and increases the instrument reliability. It should be stressed that due to the reduced available mass and power allocations onboard the MAGION-4 subsatellite, experiments have been made as simple as possible to fulfil their scientific objectives. In accordance with this idea the instruments onboard the MAGION-4 satellite are composed of three parts:

- A set of four Faraday's cups (FCs) for the determination of the total ion-flow vector (e.g. Avanov et al., 1984).

- Two cylindrical electrostatic analysers for a precise determination of the ion velocity and temperature looking in the sunward and antisunward directions. The cylindrical analysers have a narrow entrance angle $\left(\sim 3^{\circ}\right)$ and provide a one-dimensional cut of the ion-velocity distribution. Santolik et al. (1991) showed that this does not influence the computed values of the velocity and temperature under a wide range of measurement conditions.

- Two hemispheric electrostatic analysers for the determination of the ion- and electron-energy distribution in several directions. 
This set of detectors can provide all basic hydrodynamic parameters which can be compared with more complex measurements taken onboard the main, INTERBALL-1 satellite. The simplification of the MAGION-4 devices in comparison with their analogues onboard the main satellite follows from stringent requirements on the weight and power consumption.

\subsection{Plasma-flow detector VDP-S}

For the determination of the integral flux and of the direction of the bulk velocity of incoming particles the VDP-S device is based on the simultaneous measurements of the currents of four identical FCs which are placed symmetrically on the subsatellite's surface with axes declined from the main subsatellite's axis by $\sim 45^{\circ}$. Due to the satellite in-flight orientation, the axis of the first FC is directed nearly towards the Sun. Due to the negative voltage $(-170 \mathrm{~V})$ which is supplied permanently to the suppressor grid, the VDP-S device registers the sum of all ions and electrons with an energy greater than $170 \mathrm{eV}$. The negative voltage value exceeds considerably the possible energy of the secondary electrons and photoelectrons arising under the action of the Sun's UV radiation and thus eliminates the contribution of these electrons to the total collector current. The other grids of the FC system are connected to the body of the subsatellite. The size of the collector, diameters of apertures and the distances between the grids are chosen so that the angular characteristics of the sensor have a triangular shape with a width of $=50^{\circ}$ on zero level. These characteristics are a result of the compromise between the optimal $\pm 90^{\circ}$ view angles and the sensor size.

The principal electrical schematic of the VDP-S detector is given in Fig. 2. The collector of the FC is

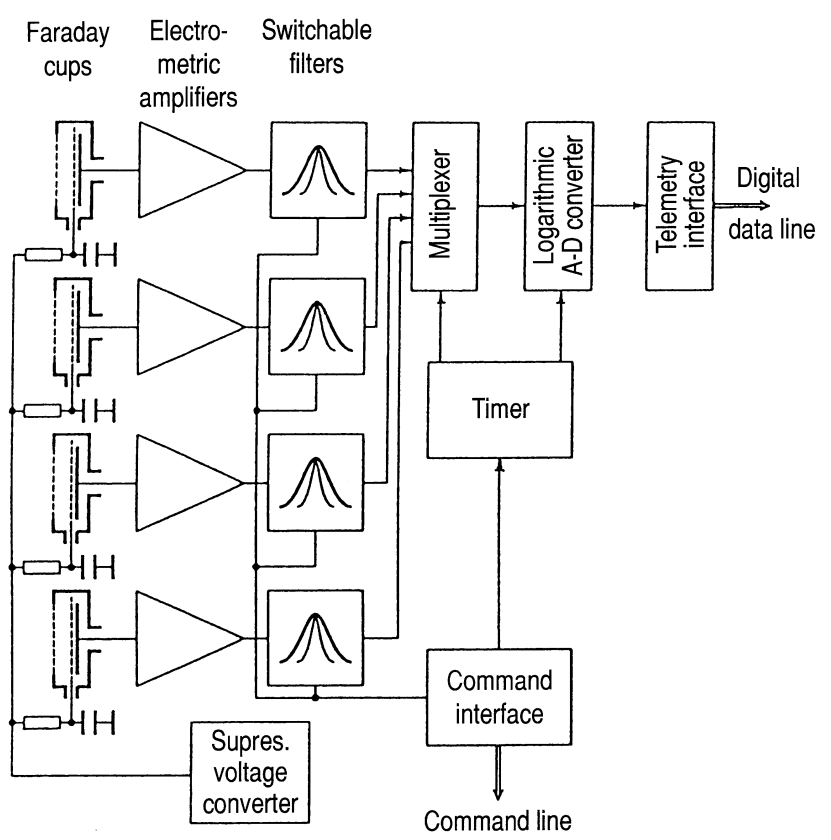

Fig. 2. Block diagram of the VDP-S device connected to the electrometric amplifier which is placed just under a particular FC to minimize the input capacitance. The amplifier output is connected to a low-pass filter. The bandwidth of the filter can be controlled from the Earth in agreement with the telemetry bit rate to keep the signal cut-off frequency in accordance with the Nyquist criterion. Filter outputs are multiplexed to the input of a logarithmic $\mathrm{AD}$ converter (ADC). The logarithmic conversion gives us the possibility to compress the polarity and the signal value with the dynamics of four orders of magnitude to eight bits with relative accuracy: $\pm 3 \%$. The frequency of the multiplexing is changed from the Earth according to the telemetry bit rate and to the number of bytes designed for the VDP-S device in the particular telemetry structure. To increase the sampling frequency by decreasing a spatial coverage, a mode in which only signals from two FCs are transmitted can be switched on via a command.

\subsection{MPS/SPS plasma spectrometers}

The MPS spectrometer was dedicated predominantly to the continuous monitoring of solar-wind parameters and for the registration of reflected ions from the bow shock. In the Earth's tail region the orientation of the MPS device along the Sun-Earth line was expected to allow the estimation of the ion leakage from the ionosphere as well as the search for counter-streaming ion beams. Due to the inclination of the actual rotational axis from the expected one, these advantages of the MPS have been lost and the MPS data can serve as a supplement to the data of the SPS spectrometer, a device which was designed for the rough determination of the angular distribution of ions and electrons and for fast measurement of their energy distribution. The angular field of view of the MPS and SPS detectors is depicted schematically in Fig. 3. One can note that the declination of the rotational axis did not change the sense of the SPS data substantially but that some part of the velocity space remained without coverage.

The MPS and SPS energy spectrometers are quite similar and most of the electronic circuits are common to both of them.

The MPS system consists of two identical sensors which are placed along the main subsatellite's axis in opposite directions. The ions come through the entrance collimator, the cylindrical electrostatic analyser and are multiplied by a channeltron. Before multiplication the ions are accelerated by the channeltron supply voltage to obtain sufficient efficiency at low energies. The schematic plot of one MPS section is given in Fig. 4.

The SPS spectrometer contains two hemispheric analysers, one for electrons, the other for ions. The particles are registered by microchannel plates (MCP) with divided collectors. This set-up has a relatively high geometric factor and allows a rough estimation of the pitch-angle distribution. From the plot in Fig. 5, where the electron section of the SPS device is shown, it can be seen that the electrons are accelerated by $\sim 150 \mathrm{~V}$, which 


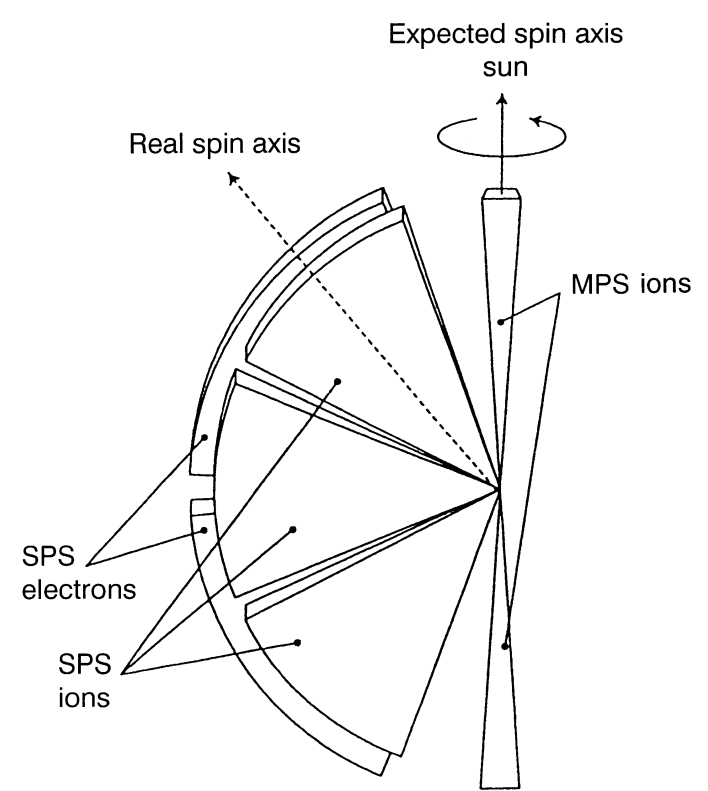

Fig. 3. Schematic plot of the MPS and SPS detectors' orientation

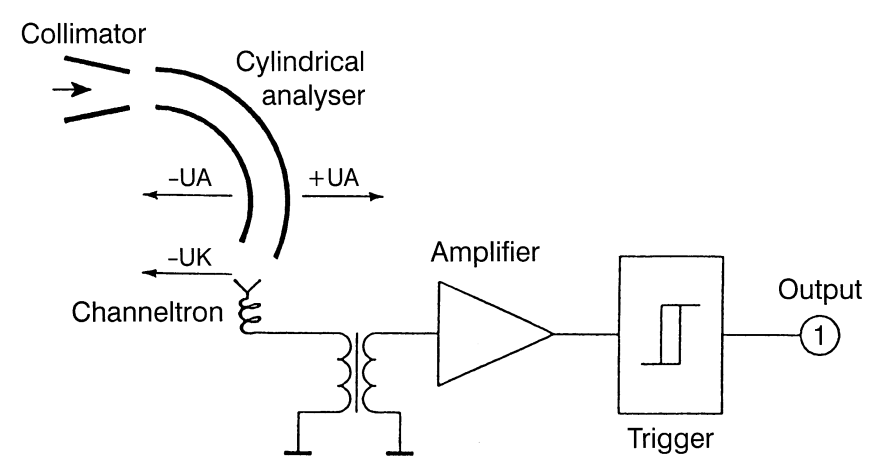

Fig. 4. Schematic plot of the MPS sensor (one section)

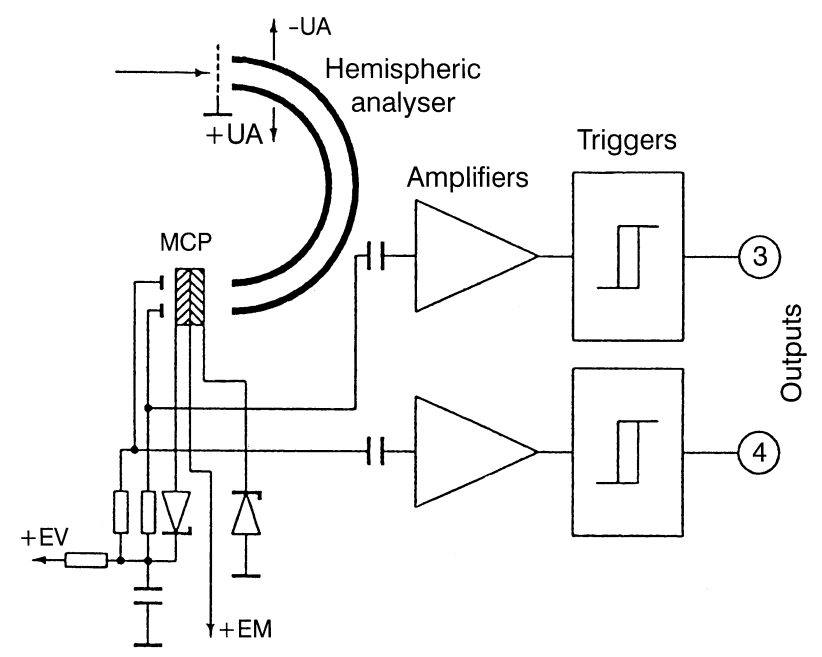

Fig. 5. Schematic plot of the electron part of the SPS sensor

is formed by the Zenner diode from the MCP supply voltage. The electrons from the MCP output are accelerated to the collector which is split into two parts. Each of these two collectors is connected to its own amplifier and Schmitt trigger. The ion section of the SPS device is nearly identical, the difference is in the MCP supply circuits and in the number of collectors only. Ions are accelerated by the full MCP supply voltage and the ion collector is divided into three parts.

The supply voltages for channeltrons and MCPs are trimmed individually and can be changed in four steps by an Earth's command independently for each detector. The analyser voltage is common to both spectrometers (SPS and MPS) and can be swept in a range of $4 \div 2500 \mathrm{~V}$. This range is divided into two subranges: $4 \div 500 \mathrm{~V}$ for solar-wind measurements and $20 \div 2500 \mathrm{~V}$ predominantly designed for magnetospheric measurements. The subrange as well as the number of steps $(4,8$ or 16) inside the subrange can be chosen by command. The ratio between the analyser voltage and the corresponding energy is $\approx 10$ for both spectrometers. The actual values of all high voltages are monitored by one multiplexed analogue telemetry channel.

Pulses from channeltrons or MCP collectors are amplified, shaped and counted by 18-bit counters. Four times per telemetry frame the counting is stopped and the logarithm of the contents of the counters is taken to obtain 8-bit output words. The results are sent to the telemetry together with the information on the current energy step.

\section{Data processing}

The actual rotational axis of the MAGION-4 satellite is declined from the expected one by about $\sim 40^{\circ}$, and this value changes from orbit to orbit. This situation changes significantly the orientation of the detectors relative to the Sun-Earth line and brings many complications in the determination of plasma parameters such as, e.g., the velocity vector. For this reason the data are presented in the satellite's frame. The data have been received during real-time transmission sessions. Due to poor quality of the transmission we had to remove all blocks with a bad check sum and this procedure caused gaps in the data. Further careful processing will allow to fill in many of these gaps.

\section{Preliminary experimental results}

Figure 6 presents the ion- and electron-energy spectrograms which have been obtained by the SPS system of the MAGION-4 satellite during 29 August 1995, from 085500 to $091500 \mathrm{UT}$. At the beginning of this timeinterval the MAGION-4 satellite was located at GSE $(X, Y, Z)=(10,-71,-11) \times 10^{3} \mathrm{~km}$ and moved nearly along the $Y$-axis towards the Earth with a velocity of 2.6 $\mathrm{km} \mathrm{s}^{-1}$. The subsatellite was followed by the INTERBALL-1 satellite separated by $1020 \mathrm{~km}$.

Three different regions denoted by $A, B$ and $C$ can be identified in the spectrograms. The $A$ region, until $085720 \mathrm{UT}$, is characterized by an intensive, nearly isotropic electron distribution (no modulation by the subsatellite's rotation can be seen). This type of distribution was observed for more than $2 \mathrm{~h}$ preceding the time-interval depicted in Fig. 6. On the other hand, 

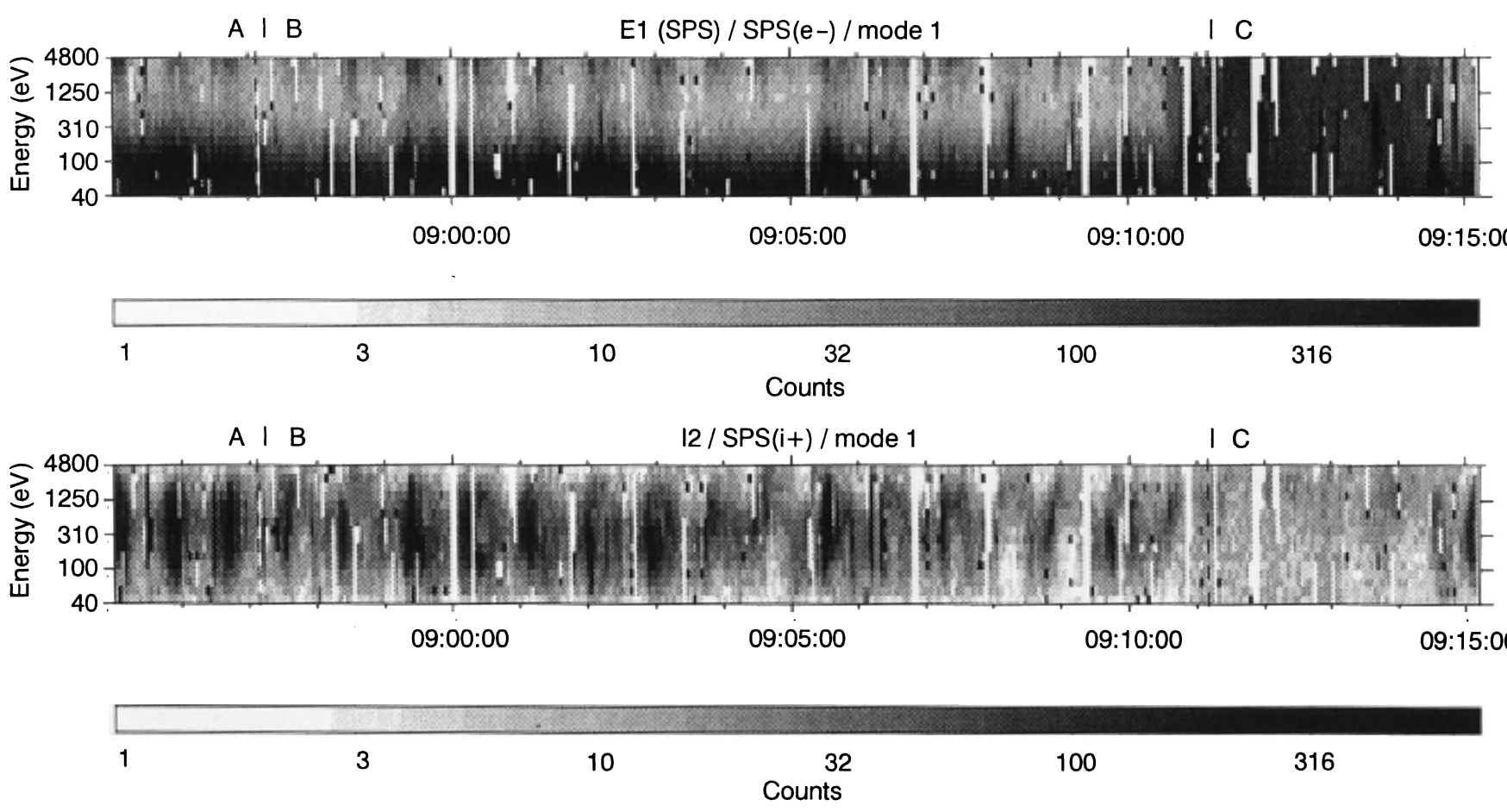

Fig. 6. Ion-and electron-energy dynamic spectrograms of the magnetopause crossing observed on 29 August by the MAGION-4 satellite. From all channels of the SPS device only those with

measurements of the ion distribution are affected by the satellite's spin. It indicates that the bulk velocity of the ion flow exceeds the thermal one. The shape of the electron and ion distributions in this region can be seen in Fig. 7a. The electrons show a typical flat-top distribution, the ion distribution exhibits a broad maximum which corresponds to a bulk velocity of about $\sim 230 \mathrm{~km} \mathrm{~s}^{-1}$. The ion temperature estimated from this distribution is about $\sim 7 \times 10^{5} \mathrm{~K}$. The shape of these energy distributions together with the spacecraft position indicate that this region is the magnetosheath.

The $B$ region starts at 085720 UT when the energy and intensity of registered electrons decreases and the ion flow nearly vanishes. The energy spectra in Fig. 7b which have been taken in this region indicate that despite the density of ions and electrons being low, the origin of the plasma is the same as in the $A$ region. Intermittent low-density and magnetosheath plasma regions are detected till 091040 UT. Energy spectra in Fig. 7c have been measured inside one of the magnetosheath intervals at 090529 UT.

The $C$ region begins at $091040 \mathrm{UT}$, when a significant increase in the electron energy is associated with the decrease in the intensity of registered ions (Fig. 7d). There can be two reasons for this decrease: (1) a real decrease in the ion density, or (2) an increase in the ion energy out of our measuring range.

The three plasma regions mentioned can be clearly distinguished in the FC data as depicted in Fig. 8. The currents are in units of the voltage on the amplifier output. This voltage is roughly proportional to the ion density through the regions $A$ and $B$ because the maximum count rate are shown. Grey scales are in counts, the counting time was $150 \mathrm{~ms}$ for each energy step

electron current is negligible in these regions and the plasma-velocity variation is of the order of $10 \%$. The FC0 (top panel) is oriented along the actual space-craft rotation axis, and thus its data are not affected by the rotation. Inside the $A$ region this FC measures the ion current higher than the upper limit of the amplifier $\left(10^{-10} \mathrm{~A}\right)$. The actual value of the input current can be estimated from FC 1 as $1.7 \times 10^{-10} \mathrm{~A}$. It corresponds to a density of about $\sim 10 \mathrm{~cm}^{-3}$. The $B$ region starts at 085720 UT by a sharp decrease in the ion current to nearly zero. This low background level is characteristic for the whole region, but above this background five sharp positive current spikes are detected. The $B$ region ends at 091030 UT when all the FCs register nearly isotropic electron current.

\section{Discussion}

The principal question in the interpretation of the data is when the magnetopause was crossed. For the crossing, we have two possible candidates: the extinction of the ion current at 085720 UT and the electron boundary at 091030 UT. The parameters of the ion flow at the beginning of the considered interval in the $A$ region are clearly those of the magnetosheath; highenergy electrons (in the $C$ region) can be attributed either to the LLBL or to the inner magnetosphere. Unfortunately, we cannot complete our observation by the reliable magnetic field measurements taken on the MAGION-4 satellite because its SGR magnetometer was designed for comparative measurements only. 

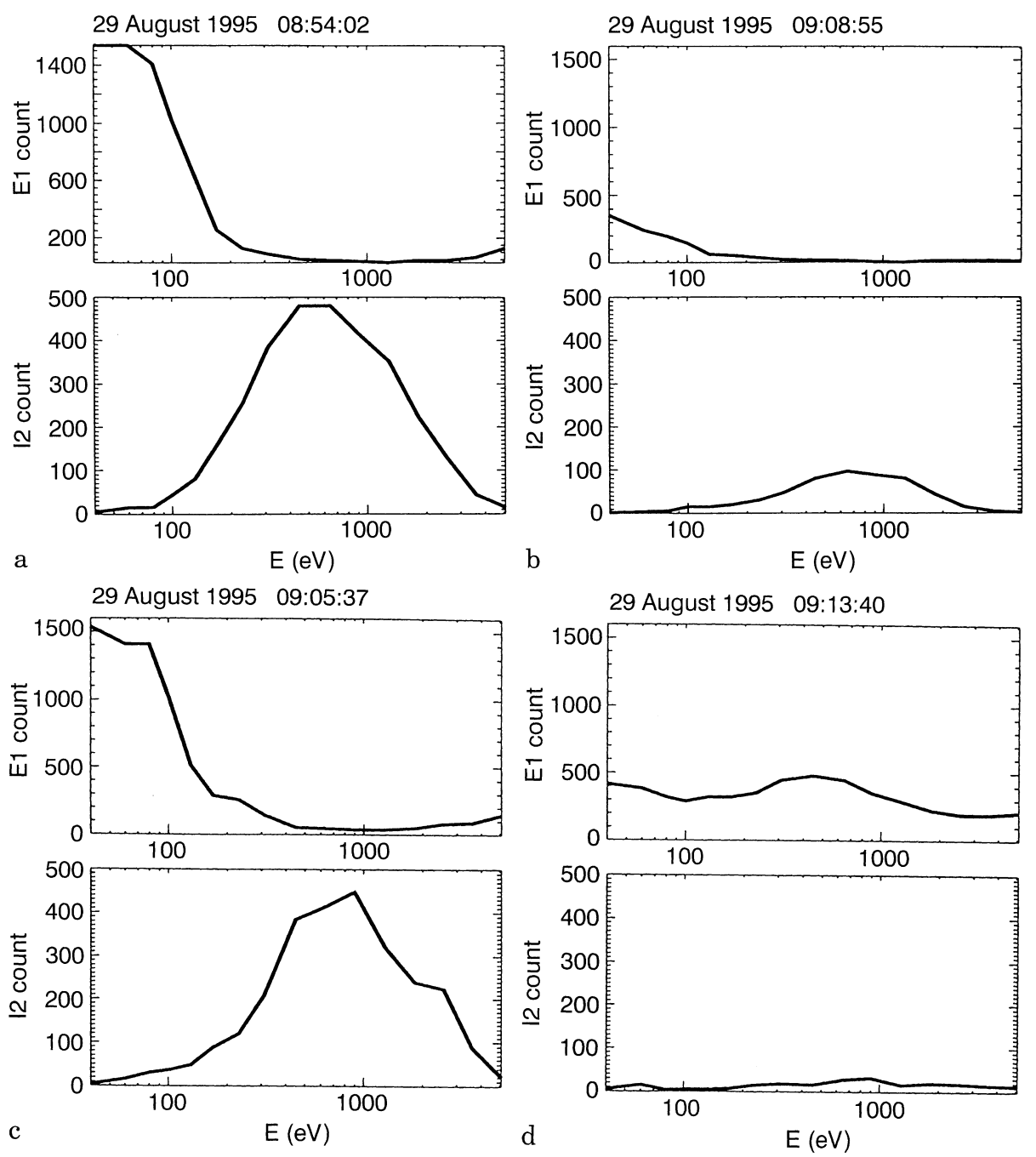

Fig. 7 a-d. Ion- and electron-energy spectra in different plasma regions (a magnetosheath, b low-density region, $\mathbf{c}$ magnetosheath-like region, d LLBL). The corresponding plasma parameters computed from the

spectra are: $\mathbf{a}-v_{1}=214 \mathrm{~km} \mathrm{~s}^{-1}, T_{i}=90 \mathrm{eV}, T_{e}=28 \mathrm{eV}, \mathbf{b} v_{i}=198 \mathrm{~km}$ $\mathrm{s}^{-1}, T_{i}=110 \mathrm{eV}, T_{e}=23 \mathrm{eV}, \mathbf{c} v_{i}=214 \mathrm{~km} \mathrm{~s}^{-1}, T_{i}=110 \mathrm{eV}, T_{e}=27$ $\mathrm{eV}, \mathbf{d} v_{i}, T_{i}-$ not determined, $T_{e}=221 \mathrm{eV}$

Moreover, the unexpected orientation of the satellite axis still did not allow to recalculate the data to the physical coordinate system. On the other hand, we can compare our data with the measurements of the INTERBALL-1, satellite which was only $1000 \mathrm{~km}$ away (Safrankova et al., 1996).

Figure 9 shows the macroscopic structure of the event. The rotation of the magnetic field together with the increase in its value occurs at $\sim 0900$ UT, the electron density completes its falling at $\sim 1025 \mathrm{UT}$. At the same time the electron temperature reaches its magnetospheric value. In accordance with Phan and Paschmann (1996), we can attribute the first boundary to the magnetopause and the second one to the inner edge of the LLBL. The considerable time-lag between these two boundaries is connected with the fact that the solar-wind pressure and the IMF were exceptionally stable during this time-interval and there was no reason for macroscopic magnetopause motion. If only the satellite velocity is taken into account, the thickness of the whole magnetopause-LLBL system is $1.6 R_{E}$.

It is necessary to note that according to the model of Sibeck et al. (1991) the expected distance of the magnetopause from the Earth under actual conditions in the solar wind measured by the WIND spacecraft (pressure $2.5 \mathrm{~Pa}, \mathrm{IMF} B_{Z} \sim-2.5 \mathrm{nT}$ between 0600 and $0900 \mathrm{UT}$ ) was $16.8 R_{E}$, but the boundary was crossed at the distance of $14.1 R_{E}$. Careful examination of the data showed that there is no indication of any crossing of any boundary between 0645 and 0850 UT.

The magnetopause crossing on a smaller scale, as measured by the INTERBALL-1 satellite, is illustrated in Fig. 10. The Figure shows the same time-interval as Figs. 6 and 8, because for this time-interval the data from both satellites are available. The magnetic field is given in the boundary normal coordinates where $B_{N}$ is taken as the minimum variance direction of the magnetic field for the period depicted in Fig. $6, B_{L}$ is the maximum variance direction in the plane perpendicular to $N$ and $B_{M}$ completes the right-hand set.

The first rotation of the magnetic field occurs at 085630 UT, when the ion density starts to fall. This 


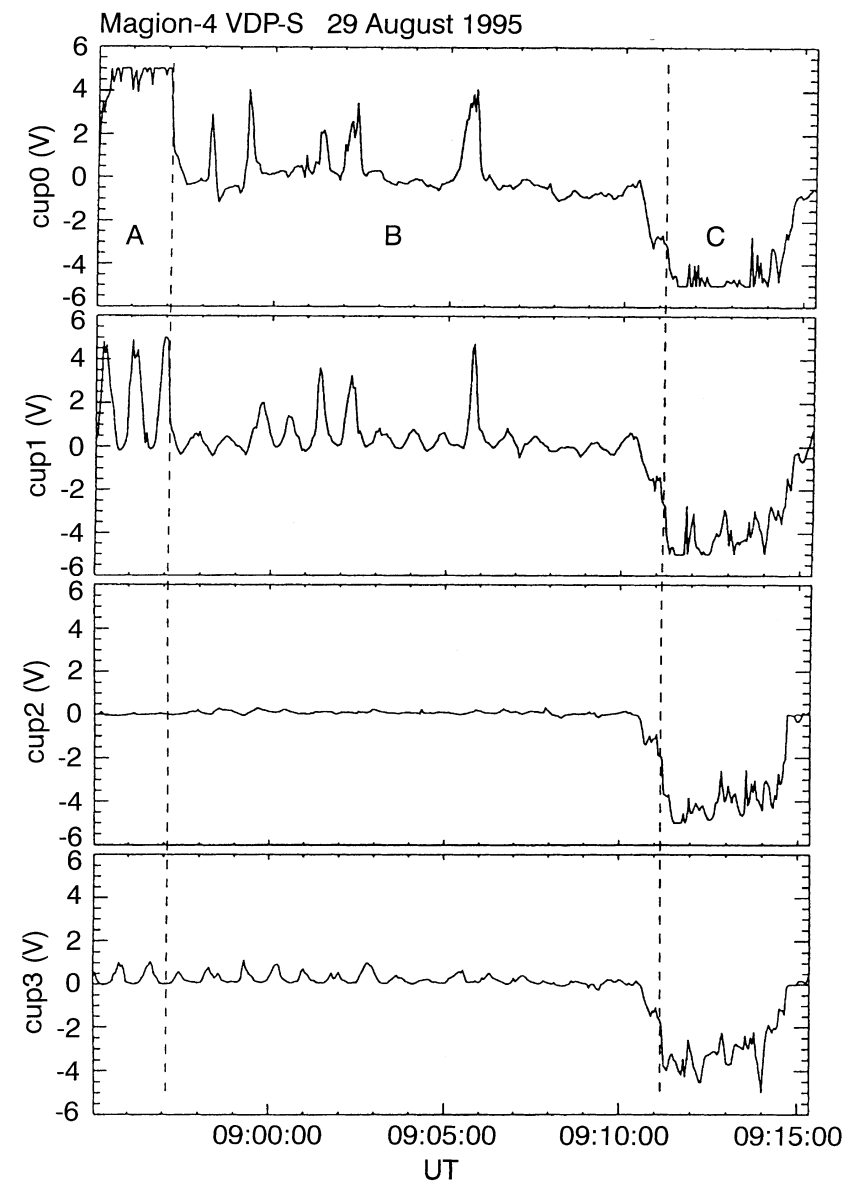

Fig. 8. Faraday's cup currents during the same event as in Fig. 6

boundary can be interpreted as the outer boundary of the plasma depletion layer. At the second distinct boundary (at 085840 UT) the magnetic field changes its value from 13 to $33 \mathrm{nT}$, but the direction remains unchanged except for short bipolar excursions of the $B_{N}$ and $B_{M}$ components, which indicate the presence of thin current layers on the boundary. The decrease in the ion density by a factor of 5 coincides with the increase in the magnetic field. We can suggest that this boundary is the magnetopause itself.

Afterwards the magnetic field and the plasma density change their values a few times nearly to the magnetosheath ones till 091015 UT. This time corresponds to the beginning of the $C$ region in Figs. 6 and 8 when the current of the FCs becomes negative (the flux of electrons with energy greater than $170 \mathrm{~V}$ becomes higher than the ion current) and thus it cannot be used for a reliable determination of the ion density. The electron density in Fig. 9 continues its decrease through the boundary and the electron temperature increases. This boundary has no signature in the magnetic field and according to Russell (1995) it can be classified as the transition to the inner part of the LLBL.

The ion-density enhancements observed afterwards can be interpreted as consecutive intensive encounters with the magnetopause because the value and fluctuations of the magnetic field are similar to those in the

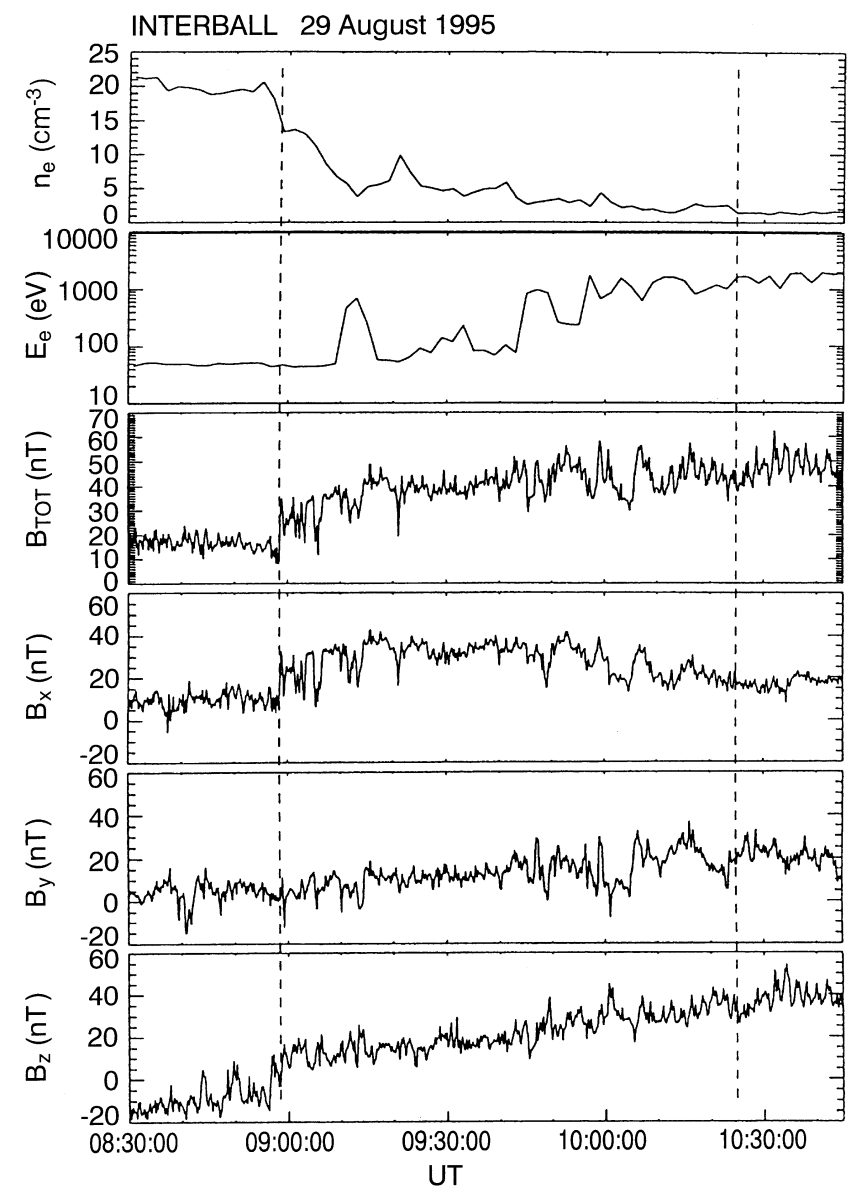

Fig. 9. Overall view of the magnetopause crossing as measured by INTERBALL-1 satellite. The dashed lines denote the magnetopause (left) and the inner edge of the LLBL (right)

magnetosheath. Similar observations of multiple iondensity enhancements made by the ISEE 2 have been interpreted as the crossing of the ion edge of the LLBL (Gosling et al., 1990). The authors suggested that the intermittent appearance of the density enhancements is caused either by the varying position of the boundary or by a non-stationary reconnection process. In our case, however, the character of the plasma inside the small regions with enhanced ion density corresponds clearly to the magnetosheath because no evidence of ion acceleration is found (Nemecek et al., 1997).

The regions of low ion and electron density around ion-density enhancements cannot be considered as belonging to the plasma depletion layer because this layer should be located outside the magnetopause. We suppose that regions are part of the LLBL, which is characterized by the permanently open field lines, and the particles which penetrate through the magnetopause are blown down. However, the origin and mechanism of the creation of the observed layer, which is characterized by $\sim 1 / 10$ of the magnetosheath plasma density and magnetospheric magnetic-field intensity, and which is located just earthward of the magnetopause, remain unclear.

The quasi-periodic appearance of the magnetosheath-like plasma here is probably caused either by a wavy magnetopause motion, similar to that depicted in 


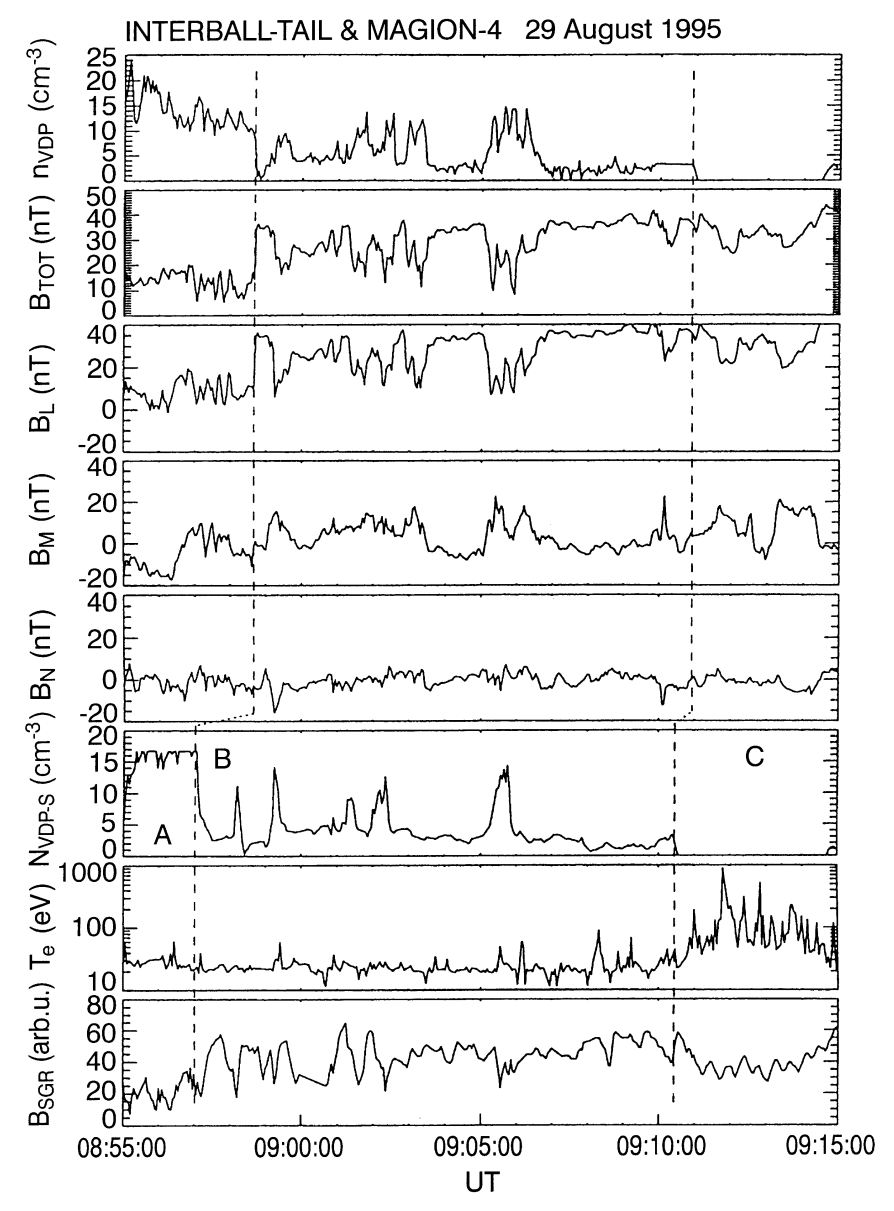

Fig. 10. Comparison of the plasma parameters and magnetic field measured by the INTERBALL 1 and MAGION-4 satellites

Fig. 1, or by flux ropes which connect the reconnection site at the magnetopause with the magnetosphere. Sibeck et al. (1990) noted that the signatures of these two cases are similar and a clear distinction can be made only after further data processing. Since the density enhancements are inside the depleted region, which probably lies on the permanently open field lines, a wavy motion of the boundary seems to be preferable for the interpretation.

The inner layer populated by hot electrons has been identified as the LLBL because the broad energy spectra are similar to those observed in the cleft region. In this case we can suppose that the energy of ions exceeds our measuring range, which is consistent with the observations presented in Fig. 6.

\section{Conclusion}

The paper presents the first results of the plasma measurements in the magnetopause region carried out by the MAGION-4 satellite. Preliminary processing of the data leads to the conclusion that the boundary layer located earthwards of the magnetopause consists of two regions: a low-density layer of the magnetopause, and the low-latitude boundary layer.
Despite the IMF being oriented southward, the crossing under study has all features reported by Russell (1995) for the cases with the northward IMF. We suggest that the classification of the crossings according the IMF- $B_{Z}$ direction can be used only for the dayside magnetopause where the magnetic field inside the LLBL has northward orientation. The local time in our case was about six o'clock and the magnetic field inbound of the magnetopause was oriented nearly along the $X_{G S E}$ axis. For such a case, the classification according to the magnetic shear is more appropriate.

Further processing of the MAGION-4 data together with the data from the INTERBALL-1 satellite can probably provide the means for a better understanding of this event, as these satellites were separated only by about $\sim 1000 \mathrm{~km}$ in the direction nearly perpendicular to the magnetopause surface during this particular magnetopause crossing.

Acknowledgements. The help of a number of colleagues who contributed to the VDP-S and MPS/SPS proposals and to the preparation of the devices is gratefully acknowledged. The last stages of the experiment preparation have been supported by the Czech Grant Agency under Contracts 205/96/1575 and 202/94/ 0467. The development and construction of the MAGION-4 satellite and its scientific payload have been supported by the Czech Grant Agency under Contract 102/93/0882. Authors express their thanks to M. Ciobanu for supplying the SGR magnetic field data.

Topical Editor K.-H. Glaßmeier thanks J. Büchner and J. Sauvaud for their help in evaluating this paper.

\section{References}

Avanov, L. A., G. N. Zastenker, O. L. Vaisberg, Yw. I. Yermolaev, Observation of small-scale solar-wind structure during the sharp increase of plasma flow velocity (in Russian) Kosmich. lssl., 22, 774, 1984.

Avanov, L. A., A. Leibov, Z. Nemecek, J. Safrankova, O. Vaisberg, J. Yermolaev, G. Zastenker, Fast measurements of solar-wind parameters by the MONITOR instrument, in: INTERSHOCK project, Ed. S. Fischer, Public. of AI CAS, 60, 39, 1985.

Bedrikov, A., A. Belikova, A. Fedorov, V. Fuchs, V. Hanzal, V. Kuzmin, A. Leibov, S. Namestnik, Z. Nemecek, V. Notkin, M. Richter, J. Safrankova, O. Vaisberg, J. Yermolaev, G. Zastenker, Complex of plasma spectrometers BIFRAM, in: INTERSHOCK project, Ed. by S. Fischer, Public. of AI CAS, 60, 113, 1985.

Cowley, S. W. H., The causes of convection in the Earth's magnetosphere: A review of developments during the IMF, Rev. Geophys., 20, 531, 1982.

Cowley, S. W. H., Solar-wind control of magnetospheric convection. Achievements of the International Magnetospheric Study IMS, ESA SP-217, ESTEC, Noordwijk, Netherlands, 483, 1984

Cowley, S. W. H., The impact of recent observations on theoretical understanding of solar wind-magnetosphere interaction. $J$. Geomagn. Geoelectr., 38, 1223, 1986.

Gosling, J. T., M. F. Thomsen, S. J. Bame, and T. G. Onsager, The electron edge of the low-latitude boundary layer during accelerated flow events, Geophys. Res. Lett., 17, 1833, 1990.

Landau, L. D., and E. M. Lifshitz, Fluid Mechanics, Pergamon, New York, 1959

Nemecek, Z., J. Safrankova, O. Santolik, K. Kudela, E. T. Sarris, Energetic particles in the vicinity of the dawn magnetopause, Adv. Space Res., in print, 1997.

Paschmann, G., G. Haerendel, I. Papamastorakis, N. Sckopke, S. J. Bame, J. T. Gosling, and C. T. Russell, Plasma and magnetic 
field characteristics of magnetic flux transfer events, J. Geophys. Res., 87, 2159, 1982.

Phan, T. D., and G. Paschmann, Low-latitude dayside magnetopause and boundary layer for high magnetic shear, 1. Structure and motion, J. Geophys, Res., 101, 7801, 1996.

Potemra, T. A., H. Luehr, L. J. Zanetti, K. Takahashi, R. E. Erlandson, G. T. Marklund, L. P. Block, L. G. Blomberg, and R. P. Lepping, Multisatellite and ground-based observations of transient ULF waves, J. Geophys. Res., 94, 2543, 1989.

Russell, C. T., The structure of the magnetopause, in Physics of the Magnetopause, ed. P. Song et al. Geophysical Monograph, 90, 81-98, 1995.

Russell, C. T., and R. C. Elphic, Initial ISEE magnetometer results: Magnetopause observation, Space Sci. Rev., 22, 681, 1978.

Safrankova, J., G. Zastenker, Z. Nemecek, A. Fedorov, M. Simersky, and L. Prech, Small-scale observation of the magnetopause motion: Preliminary results of the INTERBALL project, this issue, 1996.

Santolik, O., J. Safrankova, and Z. Nemecek, The method of thermodynamics calculation and its application on the study of protons and alpha particles behaviour in the bow shock, Czech, J. Phys., B 41, 381, 1991.

Saunders, M. A., Recent ISEE observations of the magnetopause and low-latitude boundary layer: A review, J. Geophys., 52, 190, 1983.

Sckopke, N., G. Paschmann, G. Haerendel, B. U. O. Sonnerup, S.J. Bame, T. G. Forbes, E. W. Hones Jr., and C. T. Russell, Structure of the low-latitude boundary layer, J. Geophys. Res., 86, 2099, 1981.
Sibeck, D. G., Transient events in the outer magnetosphere: Boundary waves or FTE's?, J. Geophys. Res., 97, 4009, 1992.

Sibeck, D.G., Transient magnetic field signatures at high latitudes, J. Geophys. Res., 98, 243, 1993.

Sibeck, D. G., W. Baumjohann, R. C. Elphic, D. H. Fairfield, J. F. Fennell, W. B. Gail, L. J. Lanzerotti, R. E. Lopez, H. Luehr, A.T.Y. Lui, C. G. Maclennan, R. W. McEntire, T. A. Potemra, T. J. Rosenberg T. J., and K. Takahashi, The magnetospheric response to 8-min-period strong-amplitude upstream pressure variations, J. Geophys. Res., 94, 2505, 1989a.

Sibeck, D. G., W. Baumjohann W., and R. E. Lopez, Solar-wind dynamic pressure variations and transient magnetospheric signatures, Geophys. Res. Lett., 16, 13, 1989b.

Sibeck, D. G., R. P. Lepping, and A. J. Lazarus, Magnetic field line draping in the plasma depletion layer, J. Geophys. Res., 95, 2433, 1990.

Sibeck, D. G., R. E. Lopez, E. C. Roelof, Solar-wind control of the magnetopause shape, location, and motion, J. Geophys. Res., 96, 5489, 1991.

Triska, P., J. Voita, and Yu. Agafonv, The INTERRALL subsatellite S2-A and S2-T, in INTERBALL Mission and Payload, Ed. Yu. Galperin et al., CNES-IKI-RSA, 100, 1995.

Zastenker, G. N., J. I. Yermolajev, S. Pinter, Z. Nemecek, J. Safrankova, A. B. Belikova, A. V. Leibov, V. Prochorenko, A. E. Stefanovic, A. G. Bedrikov, B. T. Karimov, Solar-wind observation with high time resolution (in Russian) Kosmich. 20, 900, 1982. 